\title{
Expanded carrier screening in an infertile population: how often is clinical decision making affected?
}

\author{
Jason M. Franasiak, MD ${ }^{1,2}$, Meir Olcha, MD ${ }^{1,2}$, Paul A. Bergh, MD², Kathleen H. Hong, MD ${ }^{1,2}$, \\ Marie D. Werner, MD ${ }^{1,2}$ Eric J. Forman, MD, HCLD ${ }^{1,2}$, Rebekah S. Zimmerman, PhD ${ }^{3}$ \\ and Richard T. Scott Jr, MD, HCLD ${ }^{1,2}$
}

Purpose: Options for preconception genetic screening have grown dramatically. Expanded carrier screening (ECS) now allows for determining carrier status for hundreds of genetic mutations by using a single sample, and some recommend ECS prior to in vitro fertilization. This study seeks to evaluate how often ECS alters clinical management when patients present for infertility care.

Methods: All patients tested with ECS at a single infertility care center from 2011 to 2014 were evaluated. The overall rate of positive ECS results and the number of couples who were carriers of the same genetic disorder were evaluated.

Results: A total of 6,643 individuals were tested, representing 3,738 couples; $1,666(25.1 \%)$ of the individuals had a positive test result for at least one disorder. In 8 of the 3,738 couples, both members of the couple were positive for the same genetic disorder or had a test result that placed them at risk of having an affected child. Three of eight cases were cystic fibrosis. In this cohort, ECS affected clinical care eight times after 6,643 tests $(0.12 \%$, confidence interval: $0.05-0.24 \%)$ in 3,738 couples $(0.21 \%$, confidence interval: $0.09-0.42 \%)$.

Conclusions: ECS is becoming more widespread. In a large case series, ECS affected clinical decision making for patients presenting for infertility care in $0.21 \%$ of cases. This information must be weighed when utilizing these tests and may be a helpful part of patient counseling.

Genet Med advance online publication 3 March 2016

Key Words: expanded carrier screening; in vitro fertilization; preimplantation genetic diagnosis

\section{INTRODUCTION}

Preconception genetic testing and counseling are important for patients undergoing infertility care. This unique population allows for thorough counseling and the opportunity to test for a variety of inheritable diseases prior to conception when in vitro fertilization and preimplantation genetic diagnosis (PGD) are used. ${ }^{1}$ If couples at risk of having an affected child are identified, then additional options for genetically screening embryos for the disease(s) prior to embryo transfer can be discussed and offered.

Traditional preconception genetic counseling and screening focus on a limited number of genes and diseases. Recommendations for testing are guided by ethnic background, severity of the disease, population carrier frequency, ability to accurately detect the disease, and significantly reduced residual risk after screening. ${ }^{2-4}$ The number of diseases that could be screened has been limited by imprecise knowledge of the genetic defect, inability to reliably identify it, and the overcall cost burden of testing.

The Human Genome Project sparked the genetic revolution involving exome and whole-genome sequencing, which has led to a more complete understanding of the human genome and subsequent identification of the genetic etiology of several pediatric diseases. Subsequently, there has been a technological revolution in genetic testing platforms involving improved time and cost parameters coupled with an ever-increasing ability to test for more mutations and diseases more quickly and more economically, culminating in expanded carrier screening. ${ }^{5-7}$ These screening panels include diseases recommended for screening by current societal practice guidelines such as those of the American College of Obstetricians and Gynecologists and the American College of Medical Genetics and Genomics, on, for example, cystic fibrosis, spinal muscular atrophy, hemoglobinopathies, and Ashkenazi Jewish diseases (e.g., Tay-Sachs disease). ${ }^{8-14}$ Although there is evidence of later-onset (TaySachs disease) or variable penetrance (Gaucher disease) in some of the Ashkenazi Jewish diseases traditionally screened for, expanded panels now include even more diseases with variable penetrance, phenotypes, and ages of onset. Additionally, the expanded panels include some diseases that do not have well-defined carrier frequencies or detection rates. This makes patient counseling regarding residual risk after screening challenging for physicians and genetic counselors. ${ }^{15}$

The advent of expanded carrier screening gives the patient and provider a great deal of information that may guide intervention in the event that carrier screening identifies a potentially

${ }^{1}$ Division of Reproductive Endocrinology and Infertility, Department of Obstetrics, Gynecology and Reproductive Science, Robert Wood Johnson Medical School, Rutgers University, New Brunswick, New Jersey, USA; ${ }^{2}$ Reproductive Medicine Associates of New Jersey; ${ }^{3}$ Foundation for Embryonic Competence. Correspondence: Jason M. Franasiak (jfranasiak@rmanj.com) 
heritable condition. However, there are limited data in the literature to inform patients and clinicians during the pretesting counseling period as to how frequently using expanded carrier screening might impact actual clinical decision making in terms of identifying a condition for which testing of preimplantation embryos may be performed. ${ }^{16,17}$ To that end, we sought to provide a descriptive analysis of expanded carrier screening at a single, large-volume infertility practice in the hopes of providing valuable information to patients and clinicians with regard to reproductive risks for patients who present for infertility care and elect to undergo preconception counseling and screening.

\section{METHODS}

This study aimed to address a practical clinical question that infertility patients and providers have regarding preconception screening utilizing expanded carrier panels: how often might one expect to have results that will alter clinical management in patients presenting for infertility care?

\section{Change in clinical decision making}

A change in clinical decision making was defined as either identification of a couple in which both members are carriers for the same autosomal recessive disorder or identification of a sexlinked genetic condition in either member of the couple seeking treatment.

\section{Data collection}

The data in this study were collected with institutional review board approval. Data were retrospectively identified in the electronic medical record system of a single, large academic infertility program from January 2011 to April 2014. Expanded carrier screening was routinely offered as an option to all patients undergoing infertility evaluation during the study period. All patients who elected to complete expanded carrier screening were included in this analysis.

Patients were tested using one of the following: (i) Inheritest, which includes 97 diseases and an additional 20 ordered on the Ashkenazi Jewish descent panel (Integrated Genetics, LabCorp Specialty Testing, Westborough, MA); (ii) the Counsyl 1.0 test, which includes 102 diseases (Counsyl, South San Francisco, CA); or (iii) Counsyl 2.0, which includes targeted mutation testing for the same 102 diseases as the 1.0 test plus sequencing to maximize coverage across genes (Counsyl). Standard practice was to order the full Inheritest panel including the additional Ashkenazi panel for all patients. The choice of panel selection was based largely on patient insurance coverage or the amount of out-of-pocket expense.

\section{Expanded carrier screening results}

All expanded carrier screening results were reviewed. First, the number of individual patient results that had at least one abnormality was identified. The results were viewed both as those for individual patients, either male or female, and also as linked couple units. The linked couple units were further analyzed to identify those tests with an abnormality that would affect clinical decision making, as defined above. Finally, the couple units with an identified abnormality that would affect clinical decision making were divided into those for which the genetic abnormality would have been identified by traditional targeted screening methods as recommended by American College of Obstetricians and Gynecologists, the traditional practice standard, and those identified only by the expanded panel. This was done because this center does have couples referred for specific disorders identified by external providers, and expanded carrier screening is then offered prior to proceeding with in vitro fertilization and PGD for the known disease state.

Proportions were calculated and the $95 \%$ confidence intervals were determined utilizing an exact binomial method.

\section{RESULTS}

\section{Expanded carrier screen tests}

During the study time frame, there were 6,643 expanded carrier tests completed. Of these, 3,577 (53.8\%) were Inheritest panels, 2,815 (42.4\%) were Counsyl v1.0 panels, and 251 (3.8\%) were Counsyl v2.0 panels. In the genes ordered, Inheritest and Counsyl 1.0 panels detected only particular known pathogenic variants, whereas Counsyl 2.0 sequenced full exonic content to detect known and novel pathogenic variants. The diseases tested for are listed in Supplementary Table S1 online.

Of the 3,738 couple units tested, 834 comprised only one patient in the couple unit. In 1,818 instances, couples underwent tandem testing with expanded carrier screening performed at the same time without waiting for test results. Finally, in 1,086 instances, sequential testing was performed in which a positive result after the first test prompted testing of the other member of the couple unit. The flow of patients and tests ordered as part of the screening paradigm are summarized in Figure 1.

\section{Patient demographics}

The mean age of the patients tested was 34.4 years (range: 21-55). Patients self-reported racial/ethnic background on an intake screening questionnaire that was reviewed and confirmed by their health-care team. Of the patients tested, 51.8\% reported their race/ethnicity as Caucasian, $14.9 \%$ as Asian,

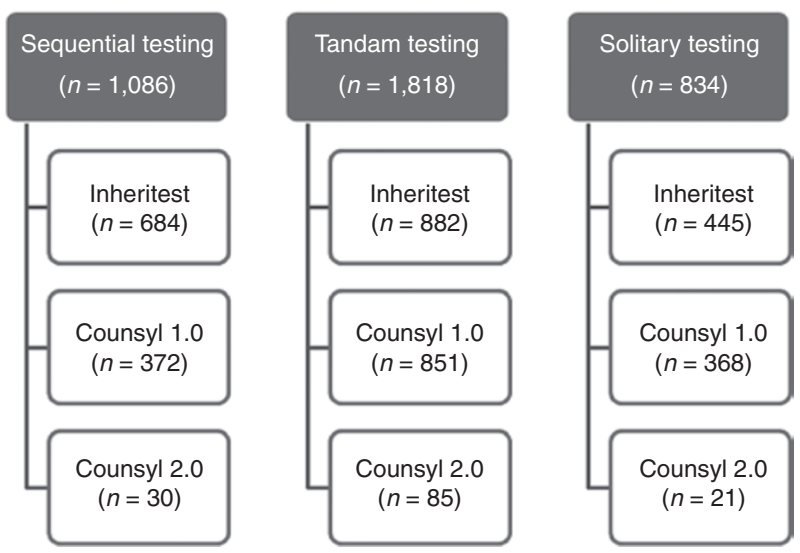

Figure 1 Flow of patient testing paradigm by couple and tests ordered. 
8.1\% as Hispanic, 6.6\% as African American, $0.1 \%$ as American Indian, $2.3 \%$ as "other," and $16.2 \%$ chose not to report.

\section{Abnormal expanded carrier screen tests}

The data were categorized in two ways: by test ordered and by couple tested. Of the 6,643 tests, 1,666 (25.1\%) had at least one abnormal result. This group of tests represented 3,738 unique couple units. In many cases only the female was tested and, if positive, testing was ordered for the male partner. Of the 1,666 positive tests, the race/ethnicity was reported as follows: $65.8 \%$ Caucasian, 8.4\% Asian, 5.5\% African American, 1.6\% “other," $0.1 \%$ American Indian, and $18.7 \%$ chose not to report.

When analyzing by couple unit, of the 3,738 couple units, there were 946 couples $(25.3 \%)$ with one or more positive results that required additional counseling. When the results of the testing for the actual mutations found within the couple unit were analyzed, there were $8(0.21 \%)$ out of 3,738 couples with mutations for the same autosomal recessive disorder in both partners or with a test result that placed them at risk of having an affected child. Three couples were found to be at risk of having a child with cystic fibrosis. The remaining four couples-all Caucasian-were at risk of having a child with carnitine palmitoyltransferase II deficiency, GJB2-related DFNB-1 nonsyndromic hearing loss, Gaucher disease, dihydrolipoamide dehydrogenase deficiency, and fragile X premutation (Table 1 ).

Fragile X disease was tested for as part of the Counsyl v1.0 and v2.0 panels, which represented less than half the panels utilized in this patient population (due to insurance preference). Among those who had the panel that included fragile $\mathrm{X}$ testing, there were three patients with intermediate (gray zone) mutations and one patient with a premutation with 60 CGG repeats, as noted above and in Table 1 .

The three cases of cystic fibrosis were already known prior to the expanded carrier screening testing. All three cystic fibrosis cases had been identified in prior pregnancies and had been referred specifically for PGD of cystic fibrosis with the hopes of transferring an unaffected, noncarrier embryo. The expanded carrier screening was performed as part of their preconception workup, and none of the couples were found to be carriers for any additional disorders. One couple had PGD testing performed but became pregnant on their own; her obstetric team ordered chorionic villus sampling. The other two couples carrying cystic fibrosis mutations did not undergo in vitro fertilization and did not pursue additional infertility treatment at this center.

Of the remaining four couples-representing cases of carnitine palmitoyltransferase II deficiency, GJB2-related DFNB-1 nonsyndromic hearing loss, Gaucher disease, and dihydrolipoamide dehydrogenase deficiency-all had preimplantation genetic testing performed on embryos prior to transfer and all four delivered unaffected babies. It is noteworthy that the couple at risk for GJB2-associated deafness, a disorder with variable penetrance, elected to undergo PGD selection to rule out an embryo affected with this disorder. While interesting, this is not uncommon in PGD testing, because historically many disorders with variable penetrance or treatment options have been screened for using PGD to reduce the burden of medical disease on the family.

In conclusion, the expanded carrier screening identified 8 (0.21\%, confidence interval: $0.09-0.42 \%)$ out of 3,738 couple units, representing 1 in 467 (confidence interval: 238-1,099) couples tested. Thus, the number of couples needed to screen to identify a couple that could be offered PGD is approximately 450. If analyzed for de novo findings, 3 of the cases of cystic fibrosis were already known; thus, de novo findings represent 1 in 748 cases.

\section{DISCUSSION}

Preconception screening utilizing expanded carrier screening is gaining acceptance from women's health-care providers, with

Table 1 Results which impacted clinical decision-making the in the couple unit undergoing expanded carrier screening as part of preconception testing

\begin{tabular}{|c|c|}
\hline Disease detected (gene: mutations) & Clinical history and overall outcomes \\
\hline Cystic fibrosis (CFTR: R553X and delta F508) & $\begin{array}{l}\text { Prior birth with CF; found at } 12 \text { weeks on routine OB screen; proceeded with pregnancy and } \\
\text { had live birth } \\
\text { PGD testing was planned but the patient became pregnant on her own and had testing by } \\
\text { chorionic villus sampling }\end{array}$ \\
\hline Cystic fibrosis (CFTR: delta F508 in both) & $\begin{array}{l}\text { Prior pregnancy with a fetus affected with CF, pregnancy was terminated } \\
\text { PGD testing was scheduled, but patient elected not to pursue treatment }\end{array}$ \\
\hline Cystic fibrosis (CFTR: c3909C>G and delta F508) & $\begin{array}{l}\text { Prior pregnancy with a fetus affected with CF, ended in a spontaneous loss } \\
\text { PGD testing was scheduled, but patient elected not to pursue treatment }\end{array}$ \\
\hline $\begin{array}{l}\text { Carnitine palmitoyltransferase II deficiency } \\
\text { (CPT2:c.1238_1239delAG (Q413fs) in both) }\end{array}$ & $\begin{array}{l}\text { New finding } \\
\text { PGD testing of embryos was performed and the patient delivered an unaffected child }\end{array}$ \\
\hline $\begin{array}{l}\text { GJB2-related DFNB-1 nonsyndromic hearing loss and } \\
\text { deficiency (GJB2: 35delG in both) }\end{array}$ & $\begin{array}{l}\text { New finding } \\
\text { PGD testing of embryos was performed and the patient delivered an unaffected child }\end{array}$ \\
\hline Gaucher disease (GBA: c.1226A>G in both) & $\begin{array}{l}\text { New finding } \\
\text { PGD testing of embryos was performed and the patient delivered an unaffected child }\end{array}$ \\
\hline $\begin{array}{l}\text { Dihydrolipoamide dehydrogenase deficiency } \\
\text { (DLD: c. } 685 \mathrm{G}>\mathrm{T} \text { in both) }\end{array}$ & $\begin{array}{l}\text { New finding } \\
\text { PGD testing of embryos was performed and the patient delivered an unaffected child }\end{array}$ \\
\hline
\end{tabular}

CF, cystic fibrosis; $\mathrm{PGD}$, preimplantation genetic diagnosis. 
more than $75 \%$ of survey providers preferring to test for more diseases, given equal cost. ${ }^{18}$ Although population prevalence data on disease frequency are known, no data exist regarding how frequently the use of expanded carrier screening might impact clinical decision making for patients presenting for infertility care. The data presented here suggest that it may be $0.21 \%$ (confidence interval: $0.09-0.42 \%$ ), indicating that $\sim 450$ couples would need to be tested to identify a disease that could then be tested for using PGD. This information is of great importance in patient counseling when discussing expanded carrier screening as part of preconception screening for infertility patients.

There are little data available that address the implementation of carrier screening protocols into real-time clinical infertility practice. Flanagan et al. reported a series of 1,669 infertile couples and the implementation of cystic fibrosis screening and spinal muscular atrophy screening into one screening package for patients. They noted an increase in utilization from 2 to $8 \%$ of couples entering care, which is an important benefit of expanding panels to include multiple diseases; however, they did not find any cases of couples at risk in this case series. ${ }^{19}$ Thus, the expansion of disease panels and finding a subset of couples subsequently eligible to undergo PGD for their single gene disorder are important.

Genetic screening paradigms are changing. Although much of genetic counseling and testing has been guided by ethnic background, this paradigm has been challenged by the everincreasing ethnic admixture of patient populations, leading some researches to regard conventional racial/ethnic labels as being of little value. ${ }^{20,21}$ This in turn has lent support to abolishing ethnicity/race-based recommendations for screening and using more comprehensive programs for universal screening. The movement toward universal screening, coupled with the reduced cost of simultaneously screening for multiple diseases, has led to changes in both expanded screening strategies and newborn screening. ${ }^{22}$

There are potential downsides to universal expanded carrier screening. Although the cost of performing the test is decreasing, the financial burden associated with counseling patients about positive results, which occurred at a rate of more than $25 \%$ in our data, has not been extensively studied. This takes health-care providers time and often involves genetic counseling for carrier states and testing of the partner at additional expense for cases that will not produce phenotypic issues in offspring because the partner is not found to also be a carrier of the same disorder.

Some limitations exist when interpreting these data. It is known that carrier frequency varies within ethnic backgrounds and therefore the prevalence of specific "at-risk" ethnicities will impact the frequency of a change in clinical decision making. Utilization of panels was not universal among all patients in this clinic. However, there is no reason to believe that patients who did not elect to have expanded carrier screening would have a higher rate of disease state than the population of patients who did elect screening.
As noted, the specific panels were chosen due to insurance coverage for patients. Hence, there was a slight difference in the diseases screened across the population according to panel. Furthermore, the majority of the panels ordered used targeted genotyping methods for expanded carrier screening. Although published data do not exist to date regarding the differences in detection when targeting genotyping and full-gene sequencing, it is plausible that this may change the frequency of positive tests as this type of technology is more widely implemented.

Panel choice as an effect of a single test can be seen with fragile $\mathrm{X}$ testing. Of the panels available to patients in this cohort, fragile X disease is included on the Counsyl v1.0 and v2.0 panels but not Inheritest. There were findings of three intermediate mutations and one premutation noted in this group, and it could be suspected that the positive test result would be higher if all patients had fragile $\mathrm{X}$ as part of their expanded carrier panel. The choice of panel for patients presenting for care was due to insurance coverage preference, as is often the case in real-world application. The purpose of this study was not to compare available platforms that might be offered but rather to explore the application of commonly used expanded carrier screening platforms in the population presenting for infertility care.

It is also important to note that this study was not intended to-nor does it have the sample size necessary to-statistically test the deviation from expected at-risk couple rates as might be seen when estimating carrier frequencies assuming HardyWeinberg equilibrium and assortive mating. Rather than an epidemiologic population-based study of genetic disease, this study focused on observation of clinical impact in a large group of infertile patients presenting to a single clinic at which expanded carrier screening is offered as an option to all patients undergoing treatment as a way of assessing how this screening paradigm has affected a large clinical cohort.

Finally, it is important to note that these data cannot be used to determine who will or will not benefit from screening. Our finding that screening would need to be performed in nearly 450 couples to impact clinical decision making does not mean that this screening does not have a very meaningful impact on a finite number of cases.

The findings presented here make an important contribution to the conversation about expanded carrier screening and its use in preconception testing of infertile patients presenting for treatment. This provides information not only to professional societies as the screening recommendations evolve but also to clinicians and, in particular, patients, who are often the ones making the decision about how to best screen for genetic diseases for which their progeny may or may not be at risk.

\section{SUPPLEMENTARY MATERIAL}

Supplementary material is linked to the online version of the paper at http://www.nature.com/gim

\section{DISCLOSURE}

The authors declare no conflict of interest. 


\section{REFERENCES}

1. American College of Obstetricians and Gynecologists Committee on Gynecologic Practice. ACOG Committee Opinion No. 452: Primary and preventive care: periodic assessments. Obstet Gynecol 2009;114:1444-51.

2. Kronn D, Jansen V, Ostrer H. Carrier screening for cystic fibrosis, Gaucher disease, and Tay-Sachs disease in the Ashkenazi Jewish population: the first 1000 cases at New York University Medical Center, New York, NY. Arch Intern Med 1998;158:777-781.

3. Dolan SM, Moore C. Linking family history in obstetric and pediatric care: assessing risk for genetic disease and birth defects. Pediatrics 2007;120(suppl 2):S66-S70.

4. Gross SJ, Pletcher BA, Monaghan KG; Professional Practice and Guidelines Committee. Carrier screening in individuals of Ashkenazi Jewish descent. Genet Med 2008; 10:54-56.

5. Srinivasan BS, Evans EA, Flannick J, et al. A universal carrier test for the long tail of Mendelian disease. Reprod Biomed Online 2010;21:537-551.

6. Bell CJ, Dinwiddie DL, Miller NA, et al. Carrier testing for severe childhood recessive diseases by next-generation sequencing. Sci Trans/ Med 2011;3:65ra4.

7. Jackson L, Pyeritz RE. Molecular technologies open new clinical genetic vistas. Sci Transl Med 2011;3:65ps2.

8. Edwards JG, Feldman G, Goldberg J, et al. Expanded carrier screening in reproductive medicine-points to consider: a joint statement of the American College of Medical Genetics and Genomics, American College of Obstetricians and Gynecologists, National Society of Genetic Counselors, Perinatal Quality Foundation, and Society for Maternal-Fetal Medicine. Obstet Gynecol 2015;125:653-662.

9. Watson MS, Cutting GR, Desnick RJ, et al. Cystic fibrosis population carrier screening: 2004 revision of American College of Medical Genetics mutation panel. Genet Med 2004;6:387-391.

10. ACOG Committee on Obstetrics. ACOG Practice Bulletin No. 78: hemoglobinopathies in pregnancy. Obstet Gynecol 2007;109:229-237.

11. ACOG Committee on Genetics. ACOG Committee Opinion No. 442: preconception and prenatal carrier screening for genetic diseases in individuals of Eastern European Jewish descent. Obstet Gynecol 2009;114:950-953.
12. ACOG Committee on Genetics. ACOG committee opinion No. 432: spinal muscular atrophy. Obstet Gynecol 2009;113:1194-1196.

13. American College of Obstetricians and Gynecologists Committee on Genetics ACOG Committee Opinion No. 486: update on carrier screening for cystic fibrosis. Obstet Gynecol 2011;117:1028-1031.

14. Prior TW; Professional Practice and Guidelines Committee. Carrier screening for spinal muscular atrophy. Genet Med 2008;10:840-842.

15. Grody WW, Thompson BH, Gregg AR, et al. ACMG position statement on prenatal/preconception expanded carrier screening. Genet Med 2013;15: 482-483.

16. Cho D, McGowan ML, Metcalfe J, Sharp RR. Expanded carrier screening in reproductive healthcare: perspectives from genetics professionals. Hum Reprod 2013;28:1725-1730

17. Martin J, Asan, Yi Y, Alberola T, Rodríguez-Iglesias B, Jiménez-Almazán J, et al. Comprehensive carrier genetic test using next-generation deoxyribonucleic acid sequencing in infertile couples wishing to conceive through assisted reproductive technology. Fertil Steril 2015;104:1286-1293.

18. Ready K, Haque IS, Srinivasan BS, Marshall JR. Knowledge and attitudes regarding expanded genetic carrier screening among women's healthcare providers. Fertil Steril 2012;97:407-413.

19. Higgins AS, Flanagan J, Hansen K, Von Wald T. Preconception CF screening in infertile couples using an expanded carrier screening test. American Congress of Obstetricians and Gynecologists Annual Clinical Meeting Chicago, IL, 26-30 April 2014.

20. Tayo BO, Teil M, Tong L, et al. Genetic background of patients from a university medical center in Manhattan: implications for personalized medicine. PLoS One 2011;6:e19166.

21. Bonham VL, Knerr S, Feero WG, Stevens $N$, Jenkins JF, McBride CM. Patient physical characteristics and primary care physician decision making in preconception genetic screening. Public Health Genomics 2010;13: 336-344.

22. Pletcher BA, Gross SJ, Monaghan KG, Driscoll DA, Watson MS. The future is now: carrier screening for all populations. Genet Med 2008;10:33-36. 